\title{
Boundedness for Commutators of Calderón-Zygmund Operator on Herz-Type Hardy Space with Variable Exponent
}

\author{
Omer Abdalrhman ${ }^{1,2}$, Afif Abdalmonem ${ }^{1,3}$, Shuangping Tao ${ }^{1}$ \\ ${ }^{1}$ College of Mathematics and Statistics, Northwest Normal University, Lanzhou, China \\ ${ }^{2}$ College of Education, Shendi University, Shendi, Sudan \\ ${ }^{3}$ Faculty of Science, University of Dalanj, Dalanj, Sudan \\ Email: "humoora@gmail.com, afeefy86@gmail.com, taop@nwnu.edu.cn
}

Received 25 May 2016; accepted 26 June 2016; published 29 June 2016

Copyright (C) 2016 by authors and Scientific Research Publishing Inc.

This work is licensed under the Creative Commons Attribution International License (CC BY).

http://creativecommons.org/licenses/by/4.0/

cc) (†) Open Access

\begin{abstract}
Our aim in this paper is to prove the boundedness of commutators of Calderón-Zygmund operator with the Lipschitz function or BOM function on Herz-type Hardy space with variable exponent.
\end{abstract}

\section{Keywords}

\section{Commutator, Variable Exponent, Herz-Taype Hardy Spaces, BMO, Calderón-Zygmund Operator}

\section{Introduction}

In 2012, Hongbin Wang and Zongguang Liu [1] discussed boundedness Calderón-Zygmund operator on Herztype Hardy space with variable exponent. M. Luzki [2] introduced the Herz space with variable exponent and proved the boundedness of some sublinear operator on these spaces. Li'na Ma, Shuhai Li and Huo Tang [3] proved the boundedness of commutators of a class of generalized Calderón-Zygmund operators on Labesgue space with variable exponent by Lipschitz function. Mitsuo Izuki [4] proved the boundedness of commutators on Herz spaces with variable exponent. Lijuan Wang and S. P. Tao [5] proved the boundedness of LittlewoodPaley operators and their commutators on Herz-Morrey space with variable exponent. In this paper we prove the boundedness of commutators of singular integrals with Lipschitz function or BMO function on Herz-type Hardy space with variable exponent.

In this section, we will recall some definitions.

Definition 1.1. Let $T$ be a singular integral operator which is initially defined on the Schwartz space $S\left(\mathbb{R}^{n}\right)$.

\footnotetext{
${ }^{*}$ Corresponding author.
}

How to cite this paper: Abdalrhman, O., Abdalmonem, A. and Tao, S.P. (2016) Boundedness for Commutators of CalderónZygmund Operator on Herz-Type Hardy Space with Variable Exponent. Journal of Applied Mathematics and Physics, 4, 1157-1167. http://dx.doi.org/10.4236/jamp.2016.46120 
Its values are taken in the space of tempered distributions $S^{\prime}\left(\mathbb{R}^{n}\right)$ such that for $x$ not in the support of $f$,

$$
\operatorname{Tf}(x)=\int_{\mathbb{R}^{n}} K(x, y) f(y) \mathrm{d} y,
$$

where $f$ is in $L_{c}^{\infty}\left(\mathbb{R}^{n}\right)$, the space of compactly bounded function.

Let $0<\delta, D<\infty$. Here the kernel $k$ is function in $\left(\mathbb{R}^{n}\right)$ away from the diagonal $x=y$ and satisfies the standard estimate

$$
|K(x, y)| \leq \frac{D}{|x-y|^{n}}, x \neq y
$$

and

$$
\left|k(x, y)-k\left(x^{\prime}, y\right)\right| \leq \frac{D\left|x-x^{\prime}\right|^{\sigma}}{|x-y|+\left|x^{\prime}-y\right|^{n+\sigma}},
$$

provided that $\left|x-x^{\prime}\right| \leq \frac{1}{2} \max \left\{|x-y|,\left|x^{\prime}-y\right|\right\}$

$$
\left|k(x, y)-k\left(x^{\prime}, y\right)\right| \leq \frac{D\left|x-x^{\prime}\right|^{\sigma}}{|x-y|+\left|x^{\prime}-y\right|^{n+\sigma}},
$$

provided that $\left|y-y^{\prime}\right| \leq \frac{1}{2} \max \left\{|x-y|,\left|x-y^{\prime}\right|\right\}$ such that is called standard kernel and the class of all kernels that satisfy (1.2), (1.3), (1.4) is denoted by $S K(\sigma, D)$. Let $T$ be as in (1.1) with kernel $S K(\sigma, D)$. If $T$ is bounded from $L^{p}$ to $L^{p}$ with $1<p<\infty$, then we say that $T$ is Calderón-Zygmund operator.

Let $\Omega$ be a measurable set in $\mathbb{R}^{n}$ with $|\Omega|>0$. We first defined Lebesgue spaces with variable exponent.

Definition 1.2. [4] Let $p(\cdot): \Omega \rightarrow[1, \infty)$ be a measurable function. The Lebesgue space with variable exponent $L^{p(\cdot)}(\Omega)$ is defined by

$$
L^{p(\cdot)}(\Omega)=\left\{f \text { is measurable }: \int_{\Omega}\left(\frac{|f(x)|}{\eta}\right)^{p(x)} \mathrm{d} x<\infty \text { for some constant } \eta>0\right\} .
$$

The space $L_{L o c}^{p(\cdot)}(\Omega)$ is defined by

$$
L_{\text {Loc }}^{p(\cdot)}(\Omega)=\left\{f \text { is measurable }: f \in L^{p(\cdot)}(K) \text { for all compact } K \subset \Omega\right\} .
$$

The Lebesgue space $L^{p(\cdot)}(\Omega)$ is a Banach space with the norm defined by

$$
\|f\|_{L^{p(\cdot)}(\Omega)}=\inf \left\{\eta>0: \int_{\Omega}\left(\frac{|f(x)|}{\eta}\right)^{p(x)} \mathrm{d} x \leq 1\right\} .
$$

We denote

$$
p_{-}=\operatorname{essinf}\{p(x): x \in \Omega\}, \quad p_{+}=\operatorname{esssup}\{p(x): x \in \Omega\} .
$$

Then $\mathcal{P}(\Omega)$ consists of all $p(\cdot)$ satisfying $p_{-}>1$ and $p_{+}<\infty$.

Let $M$ be the Hardy-Littlewood maximal operator. We denote $\mathfrak{B}(\Omega)$ to be the set of all function $p(\cdot) \in$ $\mathcal{P}(\Omega)$ satisfying that $M$ is bounded on $L^{p(\cdot)}(\Omega)$.

Let $B_{k}=\left\{x \in \mathbb{R}^{n}:|x| \leq 2^{k}\right\}, C_{k}=B_{k} \backslash B_{k-1}, \chi_{k}=\chi_{B_{k}}, k \in \mathbb{Z}$.

Proposition 1.1. See [1]. If $q(\cdot) \in \mathcal{P}(\Omega)$ satisfies

$$
\begin{gathered}
|q(x)-q(y)| \leq \frac{-A}{\log (|x-y|)},|x-y| \leq 1 / 2, \\
|q(x)-q(y)| \leq \frac{A}{\log (e+|x|)},|y| \geq|x|,
\end{gathered}
$$


then, we have $q(\cdot) \in \mathfrak{B}(\Omega)$.

Proposition 1.2. [6] Suppose that $q_{1}(\cdot), q_{2}(\cdot) \in \mathcal{P}\left(\mathbb{R}^{n}\right), 0<\gamma<n /\left(q_{1}\right)_{+}$, if $\gamma / n=1 / q_{1}(\cdot)-1 / q_{2}(\cdot)$ then

$$
\left\|\chi_{B_{k}}\right\|_{L^{a_{2}(\cdot)}\left(\mathbb{R}^{n}\right)} \leq C 2^{-k \gamma}\left\|\chi_{B}\right\|_{L^{L_{1}(\cdot)}\left(\mathbb{R}^{n}\right)},
$$

for all balls $B_{k}=\left\{x \in \mathbb{R}^{n}:|x| \leq 2^{k}\right\}$ with $k \in \mathbb{Z}$.

Definition 1.3. [7] Let $\alpha \in \mathbb{R}, \quad 0<p_{1} \leq \infty$ and $q(\cdot) \in \mathcal{P}\left(\mathbb{R}^{n}\right)$. The homogeneous Herz space with variable exponent $\dot{K}_{q(\cdot)}^{\alpha, p}\left(\mathbb{R}^{n}\right)$ is defined by

$$
\dot{K}_{q(\cdot)}^{\alpha, p}\left(\mathbb{R}^{n}\right)=\left\{f \in L_{l o c}^{q(\cdot)}\left(\mathbb{R}^{n}\right):\|f\|_{\dot{K}_{q(\cdot)}^{\alpha, p} \mathbb{R}^{n}}<\infty\right\},
$$

where

$$
\|f\|_{K_{q(\cdot)}^{\alpha, p_{2}}\left(\mathbb{R}^{n}\right)}=\left\{\sum_{k=-\infty}^{\infty} 2^{k \alpha p}\left\|f \chi_{k}\right\|_{L^{q_{2}(\cdot)}\left(\mathbb{R}^{n}\right)}^{p_{1}}\right\}^{\frac{1}{p_{1}}} .
$$

The non-homogeneous Herz space with variable exponent $K_{q(\cdot)}^{\alpha, p}\left(\mathbb{R}^{n}\right)$ is defined by

$$
K_{q(\cdot)}^{\alpha, p}\left(\mathbb{R}^{n}\right)=\left\{f \in L_{l o c}^{q(\cdot)}\left(\mathbb{R}^{n}\right):\|f\|_{K_{q(\cdot)}^{\alpha, p}\left(\mathbb{R}^{n}\right)}<\infty\right\}
$$

where

$$
\|f\|_{K_{q(\cdot)}^{\alpha, p}\left(\mathbb{R}^{n}\right)}=\left\{\sum_{k=-\infty}^{\infty} 2^{k \alpha p}\left\|f \tilde{\chi}_{k}\right\|_{L^{q(\cdot)}\left(\mathbb{R}^{n}\right)}^{p}\right\}^{\frac{1}{p}} .
$$

Definition 1.4. [1] Let $\alpha \in \mathbb{R}, 0<p \leq \infty$ and $q(\cdot) \in \mathcal{P}\left(\mathbb{R}^{n}\right)$ and $N>n+1$. Suppose that $G_{N} f(x)$ is maximal function of $f$. Homogeneous variable exponent Herz-tybe Hardy spaces $H \dot{K}_{q(\cdot)}^{\alpha, P}\left(\mathbb{R}^{n}\right)$ is defined by

$$
H \dot{K}_{q(\cdot)}^{\alpha, p}\left(\mathbb{R}^{n}\right)=\left\{f \in S^{\prime}\left(\mathbb{R}^{n}\right): G_{N} f(x) \in \dot{K}_{q(\cdot)}^{\alpha, p}\left(\mathbb{R}^{n}\right)\right\},
$$

with norm

$$
\|f\|_{H K_{q(\cdot)}^{\alpha, p}\left(\mathbb{R}^{n}\right)}=\left\|G_{N} f(x)\right\|_{\dot{K}_{q(\cdot)}^{\alpha, p}\left(\mathbb{R}^{n}\right)} \cdot
$$

Definition 1.5. [1] Let $n \delta_{2} \leq \alpha<\infty, q(\cdot) \in \mathcal{P}\left(\mathbb{R}^{n}\right), \quad\left(0<\delta_{2}<1\right)$, and non negative integer $s \geq\left[\alpha-n \delta_{2}\right]$. A function $g$ on $\mathbb{R}^{n}$ is said to be a central $(\alpha, q(\cdot))$, if satisfies

1) $\operatorname{supp} g \subset B(0, r)=\left\{x \in \mathbb{R}^{n}:|x|<r\right\}$;

2) $\|g\|_{q(\cdot)\left(\mathbb{R}^{n}\right)} \leq|B(0, r)|^{\frac{-\alpha}{n}}$;

3) $\int_{\mathbb{R}^{n}} g(x) x^{\ell} \mathrm{d} x=0,|\ell| \leq s$.

What's more, when $q(\cdot) \in \mathcal{P}\left(\mathbb{R}^{n}\right)$,

$$
\|f\|_{H K_{q(\cdot)}^{\alpha, p}\left(\mathbb{R}^{n}\right)} \approx \inf \left\{\sum_{j=-\infty}^{\infty}\left|\lambda_{j}\right|^{p}\right\}^{\frac{1}{p}} .
$$

Definition 1.6. [7] $1<\gamma \leq 0$ the Lipschiz space is defined by

$$
\operatorname{Lip}_{\gamma}\left(\mathbb{R}^{n}\right)=\left\{f:\|f\|_{L i p_{\gamma}}=\sup _{x, y \in \mathbb{R}^{n} ; x \neq y} \frac{|f(x)-f(y)|}{|x-y|^{\gamma}}<\infty\right\} .
$$

Definition 1.7. For $b \in L_{\text {loc }}^{1}\left(\mathbb{R}^{n}\right)$, the bounded mean oscillation space $B M O\left(\mathbb{R}^{n}\right)$ is defined by

$$
\|b\|_{B M O\left(\mathbb{R}^{n}\right)}=\sup _{B: b a l l s \in\left(\mathbb{R}^{n}\right)} \int_{B} \frac{1}{|B|}\left|b(x)-b_{B}\right| \mathrm{d} x .
$$




\section{Main Result and Proof}

In order to prove result, we need recall some lemma.

Lemma 2.1. ([3]) Let $b \in \operatorname{lip}_{\gamma}(0<\gamma<1)$, $T$ be Calderón-Zygmund operator, $q_{1}(\cdot), q_{2}(\cdot) \in \mathcal{B}\left(\mathbb{R}^{n}\right)$, $\frac{1}{q_{1}(\cdot)}-\frac{1}{q_{2}(\cdot)}=\frac{\beta}{n}$ Then,

$$
\|[b, T]\|_{L^{q_{2}(\cdot)}\left(\mathbb{R}^{n}\right)} \leq C\|b\|_{l i p_{\gamma}}\|f\|_{L^{q_{11}(\cdot)}\left(\mathbb{R}^{n}\right)} .
$$

Lemma 2.2. ([8]) Let $q(\cdot) \in \mathcal{P}\left(\mathbb{R}^{n}\right)$; if $f \in L^{q(\cdot)}\left(\mathbb{R}^{n}\right)$ and $g \in L^{q^{\prime}(\cdot)}\left(\mathbb{R}^{n}\right)$, then

$$
\int_{\mathbb{R}^{n}}|f(x) g(x)| \mathrm{d} x \leq r_{q}\|f\|_{L^{q(\cdot)}\left(\mathbb{R}^{n}\right)}\|g\|_{L^{q}(\cdot)\left(\mathbb{R}^{n}\right)}
$$

where $r_{q}=1+\frac{1}{q_{-}}-\frac{1}{q_{+}}$.

Lemma 2.3. ([2]) Let $q(\cdot) \in \mathcal{B}\left(\mathbb{R}^{n}\right)$. Then for all ball B in $\mathbb{R}^{n}$,

$$
|B|^{-1}\left\|\chi_{B}\right\|_{L^{q(\cdot)}\left(\mathbb{R}^{n}\right)}\left\|\chi_{B}\right\|_{L^{q}(\cdot)\left(\mathbb{R}^{n}\right)} \leq C .
$$

Lemma 2.4. ([2]) Let $q_{1}(\cdot) \in \mathcal{B}\left(\mathbb{R}^{n}\right)$ then for all measurable subsets $S \subset B$, and all ball B in $\mathbb{R}^{n}$

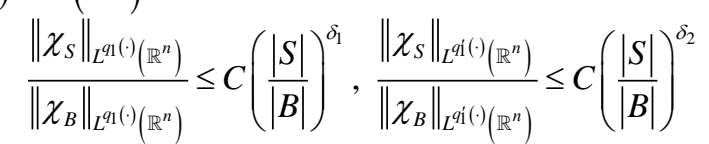

where $\delta_{1}, \delta_{2}$ are constants with $0<\delta_{1}, \delta_{2}<1$.

Lemma 2.5. ([4]) Let $b \in B M O\left(\mathbb{R}^{n}\right)$, and $i, j \in \mathbb{Z}$ with $i<j$ then

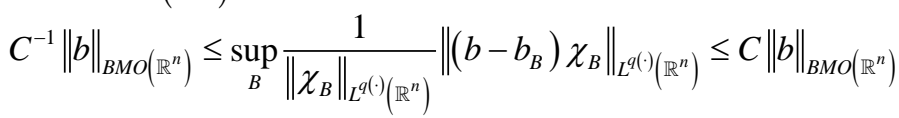

$$
\begin{aligned}
& \left\|\left(b-b_{B_{i}}\right) \chi_{B_{j}}\right\|_{L^{q(\cdot)}\left(\mathbb{R}^{n}\right)} \leq C(j-i)\|b\|_{B M O\left(\mathbb{R}^{n}\right)}\left\|\chi_{B_{j}}\right\|_{L^{q(\cdot)}\left(\mathbb{R}^{n}\right)} .
\end{aligned}
$$

Lemma 2.6. ([9]) Let $q(\cdot) \in \mathcal{B}\left(\mathbb{R}^{n}\right), b \in B M O$ function and $T$ be a Calderón-Zygmund operator. Then

$$
\|[b, T] f\|_{q(\cdot)}\left(\mathbb{R}^{n}\right) \leq C\|b\|_{B M O\left(\mathbb{R}^{n}\right)}\|f\|_{q(\cdot)}\left(\mathbb{R}^{n}\right) .
$$

Theorem 2.1. Let $q_{1}(\cdot), \quad q_{2}(\cdot) \in \mathcal{B}\left(\mathbb{R}^{n}\right), \quad b \in \operatorname{Lip}_{\gamma}, \quad 0<p_{1}<\infty, 1 / q_{1}(\cdot)-1 / q_{2}(\cdot)=\gamma / n$ and $-n \delta_{1}<\alpha<n \delta_{2}$ where $\delta_{1}, \delta_{2}$ are a constants, then $[b, T]$ are bounded from $H \dot{K}_{q_{1}(\cdot)}^{\alpha, p}\left(\mathbb{R}^{n}\right)$ to $\dot{K}_{q_{2}(\cdot)}^{\alpha, p}\left(\mathbb{R}^{n}\right)$.

Proof: we suffices to prove homogeneous case. Let $f(x) \in H \dot{K}_{q(\cdot)}^{\alpha, p}\left(\mathbb{R}^{n}\right), f=\sum_{j=-\infty}^{\infty} \lambda_{j} g_{j}$ in the $S^{\prime}\left(\mathbb{R}^{n}\right)$ sense, where each $g_{j}$ is a central $(\alpha, q(\cdot))$-atom with supp $g_{j} \subset B_{j}$. Write

We have

$$
\|f\|_{H \dot{K}_{\alpha_{1}(\cdot)}^{\alpha, p_{1}\left(\mathbb{R}^{n}\right)}} \approx \inf \left\{\sum_{j=-\infty}^{\infty}\left|\lambda_{j}\right|^{p_{1}}\right\}^{\frac{1}{p_{1}}} .
$$

$$
\begin{aligned}
& \left\|([b, T] f) \chi_{k}\right\|_{\dot{K}_{q_{2}(\cdot)}^{\alpha, p_{2}\left(\mathbb{R}^{n}\right)}}=\sum_{k=-\infty}^{\infty} 2^{k \alpha p_{1}}\left\|([b, T] f) \chi_{k}\right\|_{L^{q_{2}(\cdot)}\left(\mathbb{R}^{n}\right)} \\
& \left\|([b, T] f) \chi_{k}\right\|_{\dot{K}_{q_{2}(\cdot)}^{\alpha, p_{2}(}\left(\mathbb{R}^{n}\right)}^{p_{1}} \leq C \sum_{k=-\infty}^{\infty} 2^{k \alpha p_{1}}\left(\sum_{j=-\infty}^{k-2}\left|\lambda_{j}\right|\left\|\left([b, T] g_{j}\right) \chi_{k}\right\|_{L^{q_{2}(\cdot)\left(\mathbb{R}^{n}\right)}}\right)^{p_{1}} \\
& +C \sum_{k=-\infty}^{\infty} 2^{k \alpha p_{1}}\left(\sum_{j=k-1}^{k+1}\left|\lambda_{j}\right|\left\|\left([b, T] g_{j}\right) \chi_{k}\right\|_{L^{q_{2}(\cdot)}\left(\mathbb{R}^{n}\right)}\right)^{p_{1}} \\
& +C \sum_{k=-\infty}^{\infty} 2^{k \alpha p_{1}}\left(\sum_{j=k+1}^{\infty}\left|\lambda_{j}\right|\left\|\left([b, T] g_{j}\right) \chi_{k}\right\|_{L^{q_{2}(\cdot)}\left(\mathbb{R}^{n}\right)}\right)^{p_{1}} \\
& =F_{1}+F_{2}+F_{3}
\end{aligned}
$$


By virtue of Lemma 2.1, we can easily see that

$$
F_{2} \leq C\|b\|_{l i p_{\gamma}\left(\mathbb{R}^{n}\right)}^{p_{1}} \sum_{j=-\infty}^{\infty}\left|\lambda_{j}\right|^{p_{1}} .
$$

First we estimate $F_{1}$. For each $j \leq k-2$ and we shall get

$$
\begin{aligned}
& \left|[b, T] g_{j}\right| \leq \int_{\mathbb{R}^{n}}\left|K(x, y)(b(x)-b(y)) g_{j}(y)\right| \mathrm{d} y \\
& \leq C \int_{\mathbb{R}^{n}} \frac{\left|(b(x)-b(y)) g_{j}(y)\right|}{|x-y|^{n}} \mathrm{~d} y \\
& \leq C\|b\|_{\operatorname{lip}_{\gamma}\left(\mathbb{R}^{n}\right)} \int_{\mathbb{R}^{n}} \frac{|x-y|^{\gamma}\left|g_{j}(y)\right|}{|x-y|^{n}} \mathrm{~d} y \\
& \leq C\|b\|_{\| i p_{\gamma}\left(\mathbb{R}^{n}\right)} \int_{\mathbb{R}^{n}} \frac{\left|g_{j}(y)\right|}{|x-y|^{n-\gamma}} \mathrm{d} y \\
& \leq C\|b\|_{l i p_{\gamma}\left(\mathbb{R}^{n}\right)} 2^{-(k-2)(n-\gamma)}\left\|g_{j}\right\|_{L^{1}\left(\mathbb{R}^{n}\right)} \\
& \leq C\|b\|_{l i p_{\gamma}\left(\mathbb{R}^{n}\right)} 2^{-(k-2)(n-\gamma)}\left\|g_{j}\right\|_{L^{q_{1}(\cdot)}\left(\mathbb{R}^{n}\right)}\left\|\chi_{B_{j}}\right\|_{L^{\mathrm{q}^{(} \cdot(\cdot)}\left(\mathbb{R}^{n}\right)} \\
& \left\|\left([b, T] g_{j}\right) \chi_{k}\right\|_{\kappa_{q_{2}(\cdot)}^{\alpha, p p_{2}\left(\mathbb{R}^{n}\right)}} \leq C\|b\|_{l i p_{\beta}\left(\mathbb{R}^{n}\right)} 2^{-(k-2)(n-\gamma)}\left\|g_{j}\right\|_{L^{q_{1}(\cdot)(}\left(\mathbb{R}^{n}\right)}\left\|\chi_{B_{j}}\right\|_{L^{L^{i}(\cdot)}\left(\mathbb{R}^{n}\right)}\left\|\chi_{B_{k}}\right\|_{L^{q^{2}(\cdot)}\left(\mathbb{R}^{n}\right)}
\end{aligned}
$$

Thus by Lemma 2.3, Lemma 2.4 and Proposition 1.2, we get

$$
\begin{aligned}
& \leq C\|b\|_{l i p_{\gamma}\left(\mathbb{R}^{n}\right)} 2^{-k \gamma} 2^{-(k-2)(n-\gamma)}\left\|g_{j}\right\|_{L^{q_{1}(\cdot)}\left(\mathbb{R}^{n}\right)}\left\|\chi_{B_{j}}\right\|_{L^{q^{i}(\cdot)}\left(\mathbb{R}^{n}\right)}\left\|\chi_{B_{k}}\right\|_{L^{q_{1}(\cdot)}\left(\mathbb{R}^{n}\right)} \\
& \leq C\|b\|_{l i p_{\gamma}\left(\mathbb{R}^{n}\right)} 2^{2(n-\gamma)} 2^{-n k+k \gamma-k \gamma}\left\|g_{j}\right\|_{L^{q_{1}(\cdot)}\left(\mathbb{R}^{n}\right)}\left\|\chi_{B_{j}}\right\|_{L^{q^{i}(\cdot)}\left(\mathbb{R}^{n}\right)}\left(\mid B_{k}\left\|\chi_{B_{k}}\right\|_{L^{q_{i}(\cdot)}\left(\mathbb{R}^{n}\right)}^{-1}\right) \\
& \leq C\|b\|_{\| i p_{\gamma}\left(\mathbb{R}^{n}\right)} 2^{n k} 2^{-n k}\left\|g_{j}\right\|_{L^{q_{1}(\cdot)}\left(\mathbb{R}^{n}\right)}\left(\frac{\left\|\chi_{B_{j}}\right\|_{L^{q_{1}}(\cdot) \cdot\left(\mathbb{R}^{n}\right)}}{\left.\left\|\chi_{B_{k}}\right\|_{L^{q^{i}(\cdot)}\left(\mathbb{R}^{n}\right)}\right)}\right. \\
& \leq C\|b\|_{l i p_{\gamma}\left(\mathbb{R}^{n}\right)} 2^{(j-k) \delta_{2} n-j \alpha}
\end{aligned}
$$

When $1<p_{1}<\infty$ and $\alpha<\delta_{2} n$, by Hölder's inequality and (2.8), we calculations

$$
\begin{aligned}
& F_{1} \leq C \sum_{k=-\infty}^{\infty} 2^{k \alpha p_{1}}\left(\sum_{j=-\infty}^{k-2}\left|\lambda_{j}\right|\left\|[b, T] g_{j} \chi_{k}\right\|_{L^{q_{2}(\cdot)}\left(\mathbb{R}^{n}\right)}\right)^{p_{1}} \\
& \leq C \sum_{k=-\infty}^{\infty} 2^{k \alpha p_{1}}\left(\sum_{j=-\infty}^{k-2}\left|\lambda \lambda_{j}\right|\|b\|_{l i p_{\gamma}\left(\mathbb{R}^{n}\right)} 2^{(j-k) \delta_{2} n-j \alpha}\right)^{p_{1}} \\
& \left.\leq C\|b\|_{l i p_{\gamma}\left(\mathbb{R}^{n}\right)}^{p_{1}} \sum_{k=-\infty}^{\infty}\left(\sum_{j=-\infty}^{k-2}\left|\lambda_{j}\right|^{p_{1}} 2^{\left[(j-k)\left(\delta_{2} n-\alpha\right)\right] \frac{p_{1}}{2}}\right) \times\left(\sum_{j=-\infty}^{k-2} 2^{\left[(j-k)\left(\delta_{2} n-\alpha\right)\right]}\right]^{\frac{p_{1}^{\prime}}{2}}\right)^{\frac{p_{1}}{p_{1}^{\prime}}} \\
& \leq C\|b\|_{l i p_{\gamma}\left(\mathbb{R}^{n}\right)}^{p_{1}} \sum_{j=-\infty}^{\infty}\left|\lambda_{j}\right|^{p_{1}}\left(\sum_{k=j+2}^{\infty} 2^{\left[(j-k)\left(\delta_{2} n-\alpha\right)\right]} \frac{p_{1}}{2}\right) \\
& \leq C\|b\|_{l i p_{y}\left(\mathbb{R}^{n}\right)}^{p_{1}} \sum_{j=-\infty}^{\infty}\left|\lambda_{j}\right|^{p_{1}}
\end{aligned}
$$

where $0<p_{1} \leq 1$ by $\alpha<\delta_{2} n$, we get 


$$
\begin{aligned}
F_{1} & \leq C \sum_{k=-\infty}^{\infty} 2^{k \alpha p_{1}}\left(\sum_{j=-\infty}^{k-2}\left|\lambda_{j}\right|\left\|\left([b, T] g_{j}\right) \chi_{k}\right\|_{L^{q_{2} \cdot(\cdot)}\left(\mathbb{R}^{n}\right)}\right)^{p_{1}} \\
& \leq C \sum_{k=-\infty}^{\infty} 2^{k \alpha p_{1}}\left(\sum_{j=-\infty}^{k-2}\left|\lambda_{j}\right|\|b\|_{l i p_{\gamma}\left(\mathbb{R}^{n}\right)} 2^{(j-k) \delta_{2} n-j \alpha}\right)^{p_{1}} \\
& \leq C\|b\|_{l i p_{\gamma}\left(\mathbb{R}^{n}\right)}^{p_{1}} \sum_{k=-\infty}^{\infty} 2^{k \alpha p_{1}}\left(\sum_{j=-\infty}^{k-2}\left|\lambda_{j}\right| 2^{(j-k) \delta_{2} n-j \alpha}\right)^{p_{1}} \\
& \leq C\|b\|_{l i p_{\gamma}\left(\mathbb{R}^{n}\right)}^{p_{1}} \sum_{k=-\infty}^{\infty}\left(\sum_{j=-\infty}^{k-2}\left|\lambda_{j}\right|^{p_{1}} 2^{(j-k)\left(\delta_{2} n-\alpha\right) p_{1}}\right) \\
& \leq C\|b\|_{l i p_{\gamma}\left(\mathbb{R}^{n}\right)}^{p_{1}} \sum_{j=-\infty}^{\infty}\left|\lambda_{j}\right|^{p_{1}}\left(\sum_{k=j+2}^{\infty} 2^{(j-k)\left(\delta_{2} n-\alpha\right) p_{1}}\right) \\
& \leq C\|b\|_{l i p_{\gamma}\left(\mathbb{R}^{n}\right)}^{p_{1}} \sum_{j=-\infty}^{\infty}\left|\lambda_{j}\right|^{p_{1}}
\end{aligned}
$$

Now we estimate $F_{3}$. For each $k \geq j+2$, we shall get

$$
\begin{aligned}
& \left|[b, T] g_{j}\right| \leq \int_{\mathbb{R}^{n}}\left|K(x, y)(b(x)-b(y)) g_{j}(y)\right| \mathrm{d} y \\
& \leq C \int_{\mathbb{R}^{n}} \frac{\left|(b(x)-b(y)) g_{j}(y)\right|}{|x-y|^{n}} \mathrm{~d} y \\
& \leq C\|b\|_{\| i p_{\gamma}\left(\mathbb{R}^{n}\right)} \int_{R^{n}} \frac{|x-y|^{\gamma}\left|g_{j}(y)\right|}{|x-y|^{n}} \mathrm{~d} y \\
& \leq C\|b\|_{\operatorname{lip}_{\gamma}\left(\mathbb{R}^{n}\right)} \int_{\mathbb{R}^{n}} \frac{\left|g_{j}(y)\right|}{|x-y|^{n-\gamma}} \mathrm{d} y \\
& \leq C\|b\|_{\operatorname{lip}_{\gamma}\left(\mathbb{R}^{n}\right)} 2^{-(k-2)(n-\gamma)}\left\|g_{j}\right\|_{L^{1}\left(\mathbb{R}^{n}\right)} \\
& \leq C\|b\|_{l i p_{\gamma}\left(\mathbb{R}^{n}\right)} 2^{-(j-2)(n-\gamma)}\left\|g_{j}\right\|_{L^{q_{1}(\cdot)}\left(\mathbb{R}^{n}\right)}\left\|\chi_{B_{j}}\right\|_{L^{q^{\prime}(\cdot)}\left(\mathbb{R}^{n}\right)} \\
& \left\|\left([b, T] g_{j}\right) \chi_{k}\right\|_{\dot{K}_{q_{2}(\cdot)}^{\alpha, p_{2}\left(\mathbb{R}^{n}\right)}} \leq C\|b\|_{l i p_{\beta}\left(\mathbb{R}^{n}\right)} 2^{-(j-2)(n-\gamma)}\left\|g_{j}\right\|_{L^{q_{1}(\cdot)}\left(\mathbb{R}^{n}\right)}\left\|\chi_{B_{j}}\right\|_{L^{q^{i}(\cdot)}\left(\mathbb{R}^{n}\right)}\left\|\chi_{B_{k}}\right\|_{L^{q_{2}(\cdot)}\left(\mathbb{R}^{n}\right)}
\end{aligned}
$$

Using the Lemma 2.3 and Lemma 2.4 and Proposition 1.2, we obtain

$$
\begin{aligned}
& \leq C\|b\|_{l i p_{\gamma}\left(\mathbb{R}^{n}\right)} 2^{-j \gamma} 2^{-(j-2)(n-\gamma)}\left\|g_{j}\right\|_{L^{q_{1}(\cdot)}\left(\mathbb{R}^{n}\right)}\left\|\chi_{B_{j}}\right\|_{L^{q^{i}(\cdot)}\left(\mathbb{R}^{n}\right)}\left\|\chi_{B_{k}}\right\|_{L^{q_{1}(\cdot)}\left(\mathbb{R}^{n}\right)} \\
& \leq C\|b\|_{l i p_{\gamma}\left(\mathbb{R}^{n}\right)} 2^{2(n-\gamma)} 2^{-n j+j \gamma-j \gamma}\left\|g_{j}\right\|_{L^{q_{1}(\cdot)}\left(\mathbb{R}^{n}\right)}\left\|\chi_{B_{k}}\right\|_{L^{q_{1}(\cdot)}\left(\mathbb{R}^{n}\right)}\left(\mid B_{j}\left\|\chi_{B_{j}}\right\|_{L^{q_{1}(\cdot)}\left(\mathbb{R}^{n}\right)}^{-1}\right) \\
& \leq C\|b\|_{l i p_{y}\left(\mathbb{R}^{n}\right)} 2^{n j} 2^{-n j}\left\|g_{j}\right\|_{L^{L^{1}(\cdot)}\left(\mathbb{R}^{n}\right)}\left(\frac{\left\|\chi_{B_{k}}\right\|_{L^{q_{1}(\cdot)}\left(\mathbb{R}^{n}\right)}}{\left\|\chi_{B_{j}}\right\|_{L^{q_{1}(\cdot)}\left(\mathbb{R}^{n}\right)}}\right) \\
& \leq C\|b\|_{\operatorname{lip}_{\gamma}\left(\mathbb{R}^{n}\right)} 2^{(k-j) \delta_{1} n-j \alpha}
\end{aligned}
$$

When $1<p_{1}<\infty$ and $\alpha>-\delta_{1} n$, by Hölder's inequality and (2.12), we have 


$$
\begin{aligned}
& F_{3} \leq C \sum_{k=-\infty}^{\infty} 2^{k \alpha p_{1}}\left(\sum_{j=k+2}^{\infty}\left|\lambda_{j}\right|\left\|\left([b, T] g_{j}\right) \chi_{k}\right\|_{L^{q_{2}(\cdot)}\left(\mathbb{R}^{n}\right)}\right)^{p_{1}} \\
& \leq C \sum_{k=-\infty}^{\infty} 2^{k \alpha p_{1}}\left(\sum_{j=k+2}^{\infty}\left|\lambda_{j}\right|\|b\|_{l i p_{\gamma}\left(\mathbb{R}^{n}\right)} 2^{(k-j) \delta_{1} n-j \alpha}\right)^{p_{1}} \\
& \left.\leq C\|b\|_{l i p_{\gamma}\left(\mathbb{R}^{n}\right)}^{p_{1}} \sum_{k=-\infty}^{\infty}\left(\sum_{j=k+2}^{\infty}\left|\lambda_{j}\right|^{p_{1}} 2^{\left[(k-j)\left(\delta_{1} n+\alpha\right)\right] \frac{p_{1}}{2}}\right) \times\left(\sum_{j=k+2}^{\infty} 2^{\left[(k-j)\left(\delta_{1} n+\alpha\right)\right]}\right)^{\frac{p_{1}^{\prime}}{2}}\right)^{\frac{p_{1}}{p_{1}^{\prime}}} \\
& \leq C\|b\|_{l i p_{\gamma}}^{p_{1}}\left(\mathbb{R}^{n}\right) \sum_{j=-\infty}^{\infty}\left|\lambda_{j}\right|^{p_{1}}\left(\sum_{k=-\infty}^{j-2} 2^{\left[(k-j)\left(\delta_{1} n+\alpha\right)\right] \frac{p_{1}}{2}}\right), \\
& \leq C\|b\|_{\operatorname{lip}_{\gamma}\left(\mathbb{R}^{n}\right)}^{p_{1}} \sum_{j=-\infty}^{\infty}\left|\lambda_{j}\right|^{p_{1}}
\end{aligned}
$$

When $1<p_{1} \leq 1$ by $\alpha>-n \delta_{1}$, we have

$$
\begin{aligned}
F_{3} & \leq C \sum_{k=-\infty}^{\infty} 2^{k \alpha p_{1}}\left(\sum_{j=k+2}^{\infty}\left|\lambda_{j}\right|\left\|\left([b, T] g_{j}\right) \chi_{k}\right\|_{L^{q_{2}(\cdot)}\left(\mathbb{R}^{n}\right)}\right)^{p_{1}} \leq C \sum_{k=-\infty}^{\infty} 2^{k \alpha p_{1}}\left(\sum_{j=k+2}^{\infty}\left|\lambda_{j}\right|\|b\|_{l i p_{\gamma}\left(\mathbb{R}^{n}\right)} 2^{(k-j) \delta_{1} n-j \alpha}\right)^{p_{1}} \\
& \leq C\|b\|_{l i p_{\gamma}\left(\mathbb{R}^{n}\right)}^{p_{1}} \sum_{k=-\infty}^{\infty}\left(\sum_{j=k+2}^{\infty}\left|\lambda_{j}\right|^{p_{1}} 2^{\left[(k-j)\left(\delta_{1} n+\alpha\right)\right] p_{1}}\right) \leq C\|b\|_{l i p_{\gamma}}^{p_{1}}\left(\mathbb{R}^{n}\right) \sum_{j=-\infty}^{\infty}\left|\lambda_{j}\right|^{p_{1}}\left(\sum_{k=-\infty}^{j-2} 2^{\left[(k-j)\left(\delta_{1} n+\alpha\right)\right] p_{1}}\right) \\
& \leq C\|b\|_{l i p_{y}\left(\mathbb{R}^{n}\right)}^{p_{1}} \sum_{j=-\infty}^{\infty}\left|\lambda_{j}\right|^{p_{1}}
\end{aligned}
$$

Combining (2.10)-(2.14), we get

$$
\|[b, T] f\|_{\dot{K}_{q_{2}(\cdot)}^{\alpha, p_{2}\left(\mathbb{R}^{n}\right)}}^{p_{1}} \leq C\|f\|_{H \dot{K}_{q_{1} \cdot()}^{\alpha, p}\left(\mathbb{R}^{n}\right)}^{p_{1}} \cdot
$$

Theorem 2.2. Let $q(\cdot) \in \mathcal{B}\left(\mathbb{R}^{n}\right), \quad b \in B M O\left(\mathbb{R}^{n}\right), \quad 0<p<\infty$, and $-n \delta_{1}<\alpha<n \delta_{2}$ where $\delta_{1}, \delta_{2}>0$ are a constants, then $[b, T]$ are bounded from $H \dot{K}_{q(\cdot)}^{\alpha, p}\left(\mathbb{R}^{n}\right)$ to $\dot{K}_{q(\cdot)}^{\alpha, p}\left(\mathbb{R}^{n}\right)$.

Proof: we suffices to prove homogeneous case. Let $f(x) \in H \dot{K}_{q(\cdot)}^{\alpha, p}\left(\mathbb{R}^{n}\right), f=\sum_{j=-\infty}^{\infty} \lambda_{j} g_{j}$ in the $S^{\prime}\left(\mathbb{R}^{n}\right)$ sense, where each $g_{j}$ is a central $(\alpha, q(\cdot))$-atom with supp $g_{j} \subset B_{j}$. Write

We have

$$
\|f\|_{H \dot{K}_{q(\cdot)}^{\alpha, p}\left(\mathbb{R}^{n}\right)} \approx \inf \left\{\sum_{j=-\infty}^{\infty}\left|\lambda_{j}\right|^{p}\right\}^{\frac{1}{p}} .
$$

$$
\left\|([b, T] f) \chi_{k}\right\|_{\dot{K}_{q(\cdot)}^{\alpha, p}\left(\mathbb{R}^{n}\right)}=\sum_{k=-\infty}^{\infty} 2^{k \alpha p}\left\|([b, T] f) \chi_{k}\right\|_{L^{q(\cdot)}\left(\mathbb{R}^{n}\right)} .
$$

By inequality (2.5)we have

$$
\begin{aligned}
\left\|([b, T] f) \chi_{k}\right\|_{\kappa_{q(\cdot)}^{\alpha, p}\left(\mathbb{R}^{n}\right)}^{p} \leq & C \sum_{k=-\infty}^{\infty} 2^{k \alpha p}\left(\sum_{j=-\infty}^{k-2}\left|\lambda_{j}\right|\left\|\left([b, T] g_{j}\right) \chi_{k}\right\|_{L^{q(\cdot)}\left(\mathbb{R}^{n}\right)}\right)^{p} \\
& +C \sum_{k=-\infty}^{\infty} 2^{k \alpha p}\left(\sum_{j=k-1}^{k+1}\left|\lambda_{j}\right|\left\|\left([b, T] g_{j}\right) \chi_{k}\right\|_{L^{q(\cdot)}\left(\mathbb{R}^{n}\right)}\right)^{p} \\
& +C \sum_{k=-\infty}^{\infty} 2^{k \alpha p}\left(\sum_{j=k+1}^{\infty}\left|\lambda_{j}\right|\left\|\left([b, T] g_{j}\right) \chi_{k}\right\|_{L^{q(\cdot)}\left(\mathbb{R}^{n}\right)}\right)^{p} \\
= & F_{1}+F_{2}+F_{3}
\end{aligned}
$$

Firstly we estimate $F_{2}$ by Lemma 2.6 we can see 


$$
F_{2} \leq C\|b\|_{B M O\left(\mathbb{R}^{n}\right)}^{p} \sum_{j=-\infty}^{\infty}\left|\lambda_{j}\right|^{p}
$$

Now we consider the estimates of $F_{1}$. Note that for each $x \in A_{k}, \quad y \in A_{j}$, and $j \leq k-2$, by generalized Hölder's inequality and Lemma 2.2, we have

$$
\begin{aligned}
& \left|[b, T] g_{j}\right| \leq \int_{A_{j}}\left|K(x, y)(b(x)-b(y)) g_{j}(y)\right| \mathrm{d} y \leq C \int_{A_{j}} \frac{\left|(b(x)-b(y)) g_{j}(y)\right|}{|x-y|^{n}} \mathrm{~d} y \\
& \leq C 2^{-n k}\left|b(x)-b_{B_{j}}\right| \int_{A_{j}}\left|g_{j}(y)\right| \mathrm{d} y+\int_{A_{j}}\left|b_{B_{j}}-b(y)\right|\left|g_{j}(y)\right| \mathrm{d} y \\
& \leq C 2^{-n k}\left\|g_{j}\right\|_{L^{(\cdot)}\left(\mathbb{R}^{n}\right)}\left[\left|b(x)-b_{B_{j}}\right|\left\|\chi_{j}\right\|_{L^{q^{\prime}(\cdot)}\left(\mathbb{R}^{n}\right)}+\left\|\left(b_{B_{j}}-b\right) \chi_{j}\right\|_{L^{q^{(} \cdot()\left(\mathbb{R}^{n}\right)}}\right]
\end{aligned}
$$

Thus by Lemma 2.5 we get

$$
\begin{aligned}
& \left\|\left([b, T] g_{j}\right) \chi_{k}\right\|_{L^{q(\cdot)}\left(\mathbb{R}^{n}\right)} \\
& \leq C 2^{-n k}\left\|g_{j}\right\|_{L^{q^{(\cdot)}\left(\mathbb{R}^{n}\right)}[}\left[\left\|\left(b-b_{B_{j}}\right) \chi_{k}\right\|_{L^{(\cdot) \cdot\left(\mathbb{R}^{n}\right)}}\left\|\chi_{j}\right\|_{L^{q^{\prime}(\cdot)}\left(\mathbb{R}^{n}\right)}+\left\|\left(b_{B_{j}}-b\right) \chi_{j}\right\|_{L^{q^{(} \cdot()\left(\mathbb{R}^{n}\right)}}\left\|\chi_{k}\right\|_{L^{q(\cdot)}\left(\mathbb{R}^{n}\right)}\right] \\
& \leq C 2^{-n k}\left\|g_{j}\right\|_{L^{q(\cdot)}\left(\mathbb{R}^{n}\right)}\left[(k-j)\|b\|_{B M O\left(\mathbb{R}^{n}\right)}\left\|\chi_{k}\right\|_{L^{(\cdot)}\left(\mathbb{R}^{n}\right)}\left\|\chi_{j}\right\|_{L^{q^{(}(\cdot)}\left(\mathbb{R}^{n}\right)}+\|b\|_{B M O\left(\mathbb{R}^{n}\right)}\left\|\chi_{k}\right\|_{L^{q(\cdot)}\left(\mathbb{R}^{n}\right)}\left\|\chi_{j}\right\|_{L^{q^{\prime}(\cdot)}\left(\mathbb{R}^{n}\right)}\right] \\
& \leq C(k-j) 2^{-n k}\left\|g_{j}\right\|_{L^{(\cdot)}\left(\mathbb{R}^{n}\right)}\|b\|_{B M O\left(\mathbb{R}^{n}\right)} \times\left\|\chi_{k}\right\|_{L^{q(\cdot)}\left(\mathbb{R}^{n}\right)}\left\|\chi_{j}\right\|_{L^{q^{(\cdot)}}\left(\mathbb{R}^{n}\right)}
\end{aligned}
$$

Thus by Lemma 2.3, Lemma 2.4 and noting that $\left\|\chi_{i}\right\|_{L^{s(\cdot)}\left(\mathbb{R}^{n}\right)} \leq\left\|\chi_{B_{i}}\right\|_{L^{(\cdot)}\left(\mathbb{R}^{n}\right)}$ we get

$$
\begin{aligned}
& \leq C(k-j) 2^{-n k}\left\|g_{j}\right\|_{L^{q(\cdot)}\left(\mathbb{R}^{n}\right)}\|b\|_{B M O}\left(\mathbb{R}^{n}\right)\left\|\chi_{B_{j}}\right\|_{L^{q^{\prime}(\cdot)}\left(\mathbb{R}^{n}\right)}\left(\left|B_{k}\right|\left\|\chi_{B_{k}}\right\|_{L^{q^{(\cdot)}(\cdot)}}^{-1} \mathbb{R}^{n}\right) \\
& \leq C\|b\|_{B M O\left(\mathbb{R}^{n}\right)} 2^{n k} 2^{-n k}\left\|g_{j}\right\|_{L^{q(\cdot)}\left(\mathbb{R}^{n}\right)}\left(\frac{\left\|\chi_{B_{j}}\right\|_{L^{q^{\prime}(\cdot)}\left(\mathbb{R}^{n}\right)}}{\left.\left\|\chi_{B_{k}}\right\|_{L^{q^{\prime}} \cdot(\cdot)\left(\mathbb{R}^{n}\right)}\right)}\right. \\
& \leq C(k-j)\|b\|_{B M O\left(\mathbb{R}^{n}\right)} 2^{(j-k) n \delta_{2}-j \alpha}
\end{aligned}
$$

When $1<p<\infty$ and $\alpha<\delta_{2} n$, by Hölder's inequality and (2.17), we calculations

$$
\begin{aligned}
F_{1} & \leq C \sum_{k=-\infty}^{\infty} 2^{k \alpha p}\left(\sum_{j=-\infty}^{k-2}\left|\lambda_{j}\right|\left\|[b, T] g_{j} \chi_{k}\right\|_{L^{(\cdot)}\left(\mathbb{R}^{n}\right)}\right)^{p} \\
& \leq C \sum_{k=-\infty}^{\infty} 2^{k \alpha p}\left(\sum_{j=-\infty}^{k-2}\left|\lambda_{j}\right|(k-j)\|b\|_{B M O\left(\mathbb{R}^{n}\right)} 2^{(j-k) n \delta_{2}-j \alpha}\right)^{p} \\
& \leq C\|b\|_{B M O\left(\mathbb{R}^{n}\right)}^{p} \sum_{k=-\infty}^{\infty}\left(\sum_{j=-\infty}^{k-2}\left|\lambda_{j}\right|^{p} 2^{(k-j)\left[-n \delta_{2}+\alpha\right] \frac{p}{2}}\right) \times\left(\sum_{j=-\infty}^{k-2}(k-j)^{p^{\prime}} 2^{(k-j)\left[-n \delta_{2}+\alpha\right] \frac{p^{\prime}}{2}}\right)^{\frac{p_{1}}{p_{1}^{\prime}}} \\
& \leq C\|b\|_{B M O\left(\mathbb{R}^{n}\right)}^{p} \sum_{k=-\infty}^{\infty}\left(\sum_{j=-\infty}^{k-2}\left|\lambda_{j}\right|^{p} 2^{(k-j)\left[-n \delta_{2}+\alpha\right] \frac{p}{2}}\right) \\
& \leq C\|b\|_{B M O\left(\mathbb{R}^{n}\right)}^{p} \sum_{j=-\infty}^{\infty}\left|\lambda_{j}\right|^{p}\left(\sum_{k=j+1}^{\infty} 2^{(k-j)\left[-n \delta_{2}+\alpha\right] \frac{p}{2}}\right) \\
& \leq C\|b\|_{B M O}^{p}\left(\mathbb{R}^{n}\right) \sum_{k=-\infty}^{\infty}\left|\lambda_{j}\right|^{p}
\end{aligned}
$$


when $0<p_{1} \leq 1$ by $\alpha<\delta_{2} n$, we get

$$
\begin{aligned}
F_{1} & \leq C \sum_{k=-\infty}^{\infty} 2^{k \alpha p}\left(\sum_{j=-\infty}^{k-2}\left|\lambda_{j}\right|\left\|\left([b, T] g_{j}\right) \chi_{k}\right\|_{L^{q()}\left(\mathbb{R}^{n}\right)}\right)^{p} \\
& \leq C \sum_{k=-\infty}^{\infty} 2^{k \alpha p}\left(\sum_{j=-\infty}^{k-2}\left|\lambda_{j}\right|(k-j)\|b\|_{B M O\left(\mathbb{R}^{n}\right)} 2^{(j-k) n \delta_{2}-j \alpha}\right)^{p} \\
& \leq C\|b\|_{B M O}^{p}\left(\mathbb{R}^{n}\right) \sum_{k=-\infty}^{\infty} 2^{k \alpha p} \sum_{j=-\infty}^{k-2}\left|\lambda_{j}\right|^{p}(k-j) 2^{\left[(j-k) n \delta_{2}-j \alpha\right] p} \\
& \leq C\|b\|_{B M O\left(\mathbb{R}^{n}\right)}^{p} \sum_{k=-\infty}^{\infty}\left|\lambda_{j}\right|^{p} \sum_{j=-\infty}^{k-2}(k-j) 2^{(k-j)\left[-n \delta_{2}+\alpha\right] p} \\
& \leq C\|b\|_{B M O\left(\mathbb{R}^{n}\right)}^{p} \sum_{j=-\infty}^{\infty}\left|\lambda_{j}\right|^{p}
\end{aligned}
$$

Finally we consider the estimates of $F_{3}$. Note that for each $x \in A_{k}, \quad y \in A_{j}$, and $k \geq j+2$, by generalized Hölder's inequality and Lemma 2.2. we have

$$
\begin{aligned}
\left|[b, T] g_{j}\right| & \leq \int_{A_{j}}\left|K(x, y)(b(x)-b(y)) g_{j}(y)\right| \mathrm{d} y \\
& \leq C \int_{A_{j}} \frac{\left|(b(x)-b(y)) g_{j}(y)\right|}{|x-y|^{n}} \mathrm{~d} y \\
& \leq C 2^{-n j}\left|b(x)-b_{B_{k}}\right| \int_{A_{j}}\left|g_{j}(y)\right| \mathrm{d} y+\int_{A_{j}}\left|b_{B_{k}}-b(y)\right|\left|g_{j}(y)\right| \mathrm{d} y \\
& \leq C 2^{-n k}\left\|g_{j}\right\|_{L^{(\cdot)}\left(\mathbb{R}^{n}\right)}\left[\left|b(x)-b_{B_{k}}\right|\left|\chi_{j}\right|_{L^{q^{(}(\cdot)}\left(\mathbb{R}^{n}\right)}+\left\|\left(b_{B_{k}}-b\right) \chi_{j}\right\|_{L^{q^{(}(\cdot)}\left(\mathbb{R}^{n}\right)}\right]
\end{aligned}
$$

Thus by Proposition 1.2, and Lemma 2.5, we get

$$
\begin{aligned}
& \left\|\left([b, T] g_{j}\right) \chi_{k}\right\|_{L^{q(\cdot)}\left(\mathbb{R}^{n}\right)} \\
& \leq C 2^{-n j}\left\|g_{j}\right\|_{L^{q(\cdot)}\left(\mathbb{R}^{n}\right)}\left[\left\|\left(b-b_{B_{k}}\right) \chi_{k}\right\|_{L^{q(\cdot)}\left(\mathbb{R}^{n}\right)}\left\|\chi_{j}\right\|_{L^{q^{(}(\cdot)}\left(\mathbb{R}^{n}\right)}+\left\|\left(b_{B_{k}}-b\right) \chi_{j}\right\|_{L^{q^{(}(\cdot)}\left(\mathbb{R}^{n}\right)}\left\|\chi_{k}\right\|_{L^{q(\cdot)}\left(\mathbb{R}^{n}\right)}\right] \\
& \leq C 2^{-n j}\left\|g_{j}\right\|_{L^{(\cdot)}\left(\mathbb{R}^{n}\right)}\left[\|b\|_{B M O\left(\mathbb{R}^{n}\right)}\left\|\chi_{k}\right\|_{L^{q(\cdot)}\left(\mathbb{R}^{n}\right)}\left\|\chi_{j}\right\|_{L^{q^{\prime}(\cdot)}\left(\mathbb{R}^{n}\right)}+(j-k)\|b\|_{B M O\left(\mathbb{R}^{n}\right)}\left\|\chi_{k}\right\|_{L^{q(\cdot)}\left(\mathbb{R}^{n}\right)}\left\|\chi_{j}\right\|_{L^{q^{\prime}(\cdot)}\left(\mathbb{R}^{n}\right)}\right]
\end{aligned}
$$

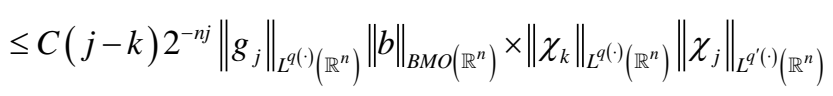

Thus by Lemma 2.3, Lemma 2.4 and noting that $\left\|\chi_{i}\right\|_{L^{5(\cdot)}\left(\mathbb{R}^{n}\right)} \leq\left\|\chi_{B_{i}}\right\|_{L^{s(\cdot)}\left(\mathbb{R}^{n}\right)}$ we get

$$
\begin{aligned}
& \leq C(j-k) 2^{-n j}\left\|g_{j}\right\|_{L^{q(\cdot)}\left(\mathbb{R}^{n}\right)}\|b\|_{B M O\left(\mathbb{R}^{n}\right)}\left\|\chi_{B_{k}}\right\|_{L^{q(\cdot)}\left(\mathbb{R}^{n}\right)}\left(\left|B_{j}\right|\left\|\chi_{B_{j}}\right\|_{L^{q \cdot(\cdot)}\left(\mathbb{R}^{n}\right)}^{-1}\right) \\
& \leq C(j-k)\|b\|_{B M O\left(\mathbb{R}^{n}\right)} 2^{n j} 2^{-n j}\left\|g_{j}\right\|_{L^{q(\cdot)}\left(\mathbb{R}^{n}\right)}\left(\frac{\left\|\chi_{B_{k}}\right\|_{L^{q(\cdot)}\left(\mathbb{R}^{n}\right)}}{\left\|\chi_{B_{j}}\right\|_{L^{q(\cdot)}\left(\mathbb{R}^{n}\right)}}\right) \\
& \leq C(j-k)\|b\|_{B M O\left(\mathbb{R}^{n}\right)} 2^{(k-j) n \delta_{1}-j \alpha}
\end{aligned}
$$

When $1<p<\infty$ and $\alpha>-\delta_{1} n$, by Hölder's inequality and (2.22), we calculations 


$$
\begin{aligned}
F_{3} & \leq C \sum_{k=-\infty}^{\infty} 2^{k \alpha p}\left(\sum_{j=k+2}^{\infty}\left|\lambda_{j}\right|\left\|[b, T] g_{j} \chi_{k}\right\|_{L^{q(\cdot)}\left(\mathbb{R}^{n}\right)}\right)^{p} \\
& \leq C \sum_{k=-\infty}^{\infty} 2^{k \alpha p}\left(\sum_{j=k+2}^{\infty}\left|\lambda_{j}\right|(j-k)\|b\|_{B M O\left(\mathbb{R}^{n}\right)} 2^{(k-j) n \delta_{1}-j \alpha}\right)^{p} \\
& \leq C\|b\|_{B M O\left(\mathbb{R}^{n}\right)}^{p} \sum_{k=-\infty}^{\infty}\left(\sum_{j=k+2}^{\infty}\left|\lambda_{j}\right|^{p} 2^{(j-k)\left[-n \delta_{2}+\alpha\right] \frac{p}{2}}\right) \times\left(\sum_{j=k+2}^{\infty}(j-k)^{p^{\prime}} 2^{(j-k)\left[-n \delta_{2}+\alpha\right] \frac{p^{\prime}}{2}}\right)^{\frac{p}{p^{\prime}}} \\
& \leq C\|b\|_{B M O\left(\mathbb{R}^{n}\right)}^{p} \sum_{k=-\infty}^{\infty}\left(\sum_{j=k+2}^{\infty}\left|\lambda_{j}\right|^{p} 2^{(j-k)\left[-n \delta_{2}+\alpha\right] \frac{p}{2}}\right) \\
& \leq C\|b\|_{B M O\left(\mathbb{R}^{n}\right)}^{p} \sum_{j=-\infty}^{\infty}\left|\lambda_{j}\right|^{p}\left(\sum_{k=\infty}^{j-2} 2^{(j-k)\left[-n \delta_{2}+\alpha\right] \frac{p}{2}}\right) \\
& \leq C\|b\|_{B M O\left(\mathbb{R}^{n}\right)}^{p} \sum_{j=-\infty}^{\infty}\left|\lambda_{j}\right|^{p}
\end{aligned}
$$

when $0<p \leq 1$ by $\alpha>-\delta_{1} n$, we get

$$
\begin{aligned}
F_{3} & \leq C \sum_{k=-\infty}^{\infty} 2^{k \alpha p}\left(\sum_{j=k+2}^{\infty}\left|\lambda_{j}\right|\left\|\left([b, T] g_{j}\right) \chi_{k}\right\|_{L^{q(\cdot)}\left(\mathbb{R}^{n}\right)}\right)^{p} \\
& \leq C \sum_{k=-\infty}^{\infty} 2^{k \alpha p}\left(\sum_{j=k+2}^{\infty}\left|\lambda_{j}\right|\|b\|_{B M O\left(\mathbb{R}^{n}\right)} 2^{(k-j) n \delta_{1}-j \alpha}\right)^{p} \\
& \leq C\|b\|_{B M O}^{p}\left(\mathbb{R}^{n}\right) \sum_{k=-\infty}^{\infty}\left(\sum_{j=k+2}^{\infty}\left|\lambda_{j}\right| 2^{(j-k)\left[-n \delta_{1}-\alpha\right]}\right)^{p} \\
& \leq C\|b\|_{B M O\left(\mathbb{R}^{n}\right)}^{p} \sum_{j=-\infty}^{\infty}\left|\lambda_{j}\right|^{p}\left(\sum_{k=\infty}^{j-2} 2^{(j-k)\left[-n \delta_{1}-\alpha\right] p}\right) \\
& \leq C\|b\|_{B M O\left(\mathbb{R}^{n}\right)}^{p} \sum_{j=-\infty}^{\infty}\left|\lambda_{j}\right|^{p}
\end{aligned}
$$

combining (2.14)-(2.24) the prove is completed.

\section{Acknowledgements}

This paper is supported by National Natural Foundation of China (Grant No. 11561062).

\section{References}

[1] Wang, H.B. and Liu, Z.G. (2012) The Herz-Type Hardy Space with Variable Exponent and Their Applications. Taiwanese Journal of Mathematics, 16, 1363-1389.

[2] Izuki, M. (2010) Boundedness of Sublinear Operators on Herz Spaces with Variable Exponent and Application to Wavelet Characterization. Analysis Mathematica, 36, 33-50. http://dx.doi.org/10.1007/s10476-010-0102-8

[3] Ma, L.N., Li, S.H. and Tang, H. (2012) Boundedness of Commutators of a Class of Generalized Calderón-Zygmund Operators on Labesgue Space with Variable Exponent. Pure Mathematics, 2, 78-81. http://dx.doi.org/10.12677/pm.2012.22013

[4] Izuki, M. (2010) Boundedness of Commutators on Herz Spaces with Variable Exponent. Rendiconti del Circolo Matematico di Palermo, 59, 199-213. http://dx.doi.org/10.1007/s12215-010-0015-1

[5] Wang, L.J. and Tao, S.P. (2014) Boundedness of Littlewood-Paley Operators and Their Commutators on Herz-Morrey Space with Variable Exponent. Journal of Inequalities and Applications, 227, 1-17. http://dx.doi.org/10.1186/1029-242x-2014-227

[6] Capone, C., Cruz-Uribe, D. and Fioenza, A. (2007) The Fractional Maximal Operator and Fractional Integerals on Va- 
riable in $L^{p}$ Space. Revista Matemática Iberoamericana, 23, 743-770. http://dx.doi.org/10.4171/RMI/511

[7] Stein, E.M. (1970) Singular Integral and Differentiability Properties of Functions. Princeton University Press, Princeton.

[8] Kováčik, O. and Rákosnk, J. (1991) On Spaces $L^{p(x)}$ and $W^{k, p(x)}$. Czechoslovak Mathematical Journal, 41, 592-618.

[9] Cruz-Uribe, D., Fiorenza, A., Martell, J.M. and Pérez, C. (2006) The Boundedness of Classical Operators on Variable $L^{p}$ Spaces. Annales Academiae Scientiarum Fennicae. Mathematica, 31, 239-264.

Submit or recommend next manuscript to SCIRP and we will provide best service for you:

Accepting pre-submission inquiries through Email, Facebook, Linkedin, Twitter, etc A wide selection of journals (inclusive of 9 subjects, more than 200 journals)

Providing a 24-hour high-quality service

User-friendly online submission system

Fair and swift peer-review system

Efficient typesetting and proofreading procedure

Display of the result of downloads and visits, as well as the number of cited articles

Maximum dissemination of your research work

Submit your manuscript at: http://papersubmission.scirp.org/ 